\title{
Evidence for specificity in type 4 pilus biogenesis by enteropathogenic Escherichia coli
}

\author{
Barry P. McNamara and Michael S. Donnenberg
}

Author for correspondence: Michael S. Donnenberg. Tel: +1 410706 7560. Fax: +1 4107068700. e-mail: mdonnenb@umaryland.edu

Division of Infectious Diseases, Department of Medicine, University of Maryland School of Medicine, 10 South Pine Street, Room 900, Baltimore, MD 21201, USA

\begin{abstract}
Type 4 fimbriae (pili) are surface appendages that are expressed by many species of Gram-negative bacteria. Previous studies have demonstrated that Pseudomonas aeruginosa can express and assemble pilin subunits from several unrelated species, indicating a common mechanism for biogenesis of type 4 pili whereby structural subunits from one system may be interchanged with those of another. In this study, an isogenic mutant of enteropathogenic Escherichia coli (EPEC) was constructed containing the entire tcpA gene from Vibrio cholerae 0395, which encodes the major structural subunit of the toxincoregulated pilus (TCP), in place of bfpA, which encodes the major structural subunit of the bundle-forming pilus (BFP). Surprisingly, expression of type 4 pilin structures and the associated phenotype of bacterial autoaggregation in culture media were not observed for cells of the EPEC strain containing tcpA nor for those containing an additional mutation in $b f p F$, which otherwise is associated with a hyperfimbriate phenotype. In addition, cells of a bfpA mutant EPEC strain containing plasmids designed to express either of two different chimeric type 4 pilin subunits containing segments of BfpA and TcpA also failed to form bacterial aggregates and express type 4 pilin structures. Collectively, these results indicate that the type 4 pilin assembly system of EPEC exhibits specificity with regard to pilin subunit recognition and assembly.
\end{abstract}

Keywords: fimbriae, bundle-forming pilus (BFP), enteropathogenic E. coli, toxincoregulated pilus (TCP), type 4 pili

\section{INTRODUCTION}

Type 4 fimbriae (pili) are flexible surface structures that are produced by a wide range of bacterial pathogens. These pili participate in a variety of bacterial processes that include bacterial adherence to host cells, target cell specificity, twitching motility, bacteriophage adsorption and DNA uptake. In certain cases, they have been shown to play a major role in mediating colonization of mammalian hosts by pathogenic bacteria such as Pseudomonas aeruginosa, Dichelobacter nodosus, Vibrio cholerae and enteropathogenic Escherichia coli (EPEC) (Herrington et al., 1988; Voss et al., 1996; Bieber et al., 1998).

Type 4 pili appear to be largely composed of a single structural subunit that has a number of distinctive

Abbreviations: BFP, bundle-forming pili; EAF, EPEC adherence factor; EPEC, enteropathogenic Escherichia coli; TCP, toxin-coregulated pili. features (Strom \& Lory, 1993). These features include a short positively charged leader sequence that is removed by a specific prepilin peptidase prior to filament assembly, an $\mathrm{N}$-methyl modification of the first amino acid residue of the mature polypeptide and a distinctive hydrophobic N-terminus of about 30 amino acids containing an invariant glutamic acid residue at the fifth position of the mature polypeptide. Conservation of the extremely hydrophobic N-terminal region of type 4 pilins is consistent with the hypothesis that this domain participates in subunit-subunit interactions, facilitating the assembly of individual pilin subunits into a mature filament (Parge et al., 1995). In addition, the C-terminal immunogenic region is predicted to form an intrachain disulfide bond between two conserved cysteine residues that confers protein stability (Sun et al., 1997; Zhang \& Donnenberg, 1996) and contains an epithelial-cellbinding domain (Sun et al., 1997; Hahn, 1997).

The type 4 family of pilin subunits can be divided into 
two groups based on the length of their leader sequence, the identity of the first residue of the mature protein and the genomic organization of genes encoding components required for their assembly. Group A pilins from $P$. aeruginosa (Johnson et al., 1986), Neisseria spp. (Potts \& Saunders, 1988; Meyer et al., 1984), Moraxella bovis (Marrs et al., 1985) and D. nodosus (McKern et al., 1988) are synthesized as prepilin precursors with short (six to seven amino acids), basic leader peptides that undergo endoproteolytic cleavage between invariant glycine and phenylalanine residues. Thus, they contain an $\mathrm{N}$-methylphenylalanine as the first residue of the mature protein. Genes encoding components required for group A pilin assembly reside in several different regions of the genome. In contrast, group B pilins composing toxin-coregulated pili (TCP) from $V$. cholerae (Shaw \& Taylor, 1990; McKern et al., 1988), bundle-forming pili (BFP) from EPEC (Sohel et al., 1993; Donnenberg et al., 1992), long pili (longus) and CFA/III from enterotoxigenic E. coli (ETEC) (Taniguchi et al., 1995; Girón et al., 1994), and thin pili encoded by the IncI1 conjugal plasmid R64 (Kim \& Komano, 1997) are synthesized as precursors with longer (13 to 30 amino acids) basic leader peptides that undergo endoproteolytic cleavage between an invariant glycine and a variable residue. Thus, the first amino acid of mature TcpA is methionine, and that of BfpA (bundlin) is leucine. $\mathrm{N}$-Methyl modification of the first residue of group B pilin peptides has thus far only been determined for TcpA (Shaw \& Taylor, 1990). The contiguous arrangement of genes encoding components required for group $\mathrm{B}$ pilus biogenesis is typified by the $t c p$ gene cluster of V. cholerae (Ogierman et al., 1993) and the bfp gene cluster, which resides on the large EPEC adherence factor (EAF) plasmid harboured in EPEC (Stone et al., 1996; Sohel et al., 1993).

Studies from several laboratories indicate that the assembly machinery required for type 4 pilus biogenesis is conserved. Pilin subunits from species including $D$. nodosus (Mattick et al., 1987), M. bovis (Beard et al., 1990) and N. gonorrhoeae (Hoyne et al., 1992) can be expressed and assembled on the cell surface of $P$. aeruginosa. All these studies, until recently, were performed in a hyperfimbriate strain of Pseudomonas that lacked twitching motility. This strain was later found to contain a mutation in pilT, a gene that encodes a nucleotide-binding protein that may participate in pilus retraction (Whitchurch et al., 1991). Koomey (1995) suggested that this background could remove constraints that otherwise would interfere with specific interactions between certain components of the fimbrial assembly system and pilin subunits derived from different species. However, Watson et al. (1996) have recently shown that $P$. aeruginosa lacking pilA in an otherwise wild-type background can assemble subunits from $D$. nodosus into fully functional pili, as demonstrated by restoration of both twitching motility and susceptibility to fimbrial-specific bacteriophages. Whether assembly of type 4 pili from a heterologous host is restricted to pili composed of group A subunits or is a particular trait of type 4 pilus biogenesis in $P$. aeruginosa is not known. Studies involving expression of type 4 pili in heterologous species have shown utility in the design of recombinant vaccines such as those against bovine keratoconjunctivitis (Lepper et al., 1995). By extending these applications to recombinant strains containing pilin subunits from group B, vaccine candidates could be designed against pathogens that predominantly cause gastrointestinal diseases in humans.

In this study, we sought to test whether components encoded by the $b f p$ gene cluster from EPEC can direct the assembly of functional type 4 pili encoded by $t c p A$ from $V$. cholerae O395. To do so, we constructed a derivative of EPEC in which the first gene of the $b f p$ gene cluster, $b f p A$, encoding the major structural subunit of $B F P$, was replaced with the $t c p A$ gene encoding the major structural subunit of TCP. We also examined whether a $b f p F$ mutation, like that of the homologous pilT mutation, would facilitate expression of type 4 pili encoded by $t c p A$, since cells of a $b f p F$ mutant are hyperfimbriate compared to wild-type EPEC (Bieber $e t$ al., 1998). Finally, we tested whether chimeric pilin subunits containing segments of BfpA and TcpA could be expressed and assembled in an EPEC strain containing a mutation in $b f p A$.

\section{METHODS}

Bacterial strains, plasmids and growth media. Bacterial strains and plasmids used in this study are listed in Table 1. E. coli $\mathrm{DH} 5 \alpha$ was used to maintain all plasmids except derivatives of the positive-selection suicide vector pCVD442, which were maintained in E. coli DH5 $\alpha \lambda$ pir. Luria-Bertani (LB) broth $\left(\mathrm{l}^{-1}\right.$ : $10 \mathrm{~g}$ tryptone, $5 \mathrm{~g}$ yeast extract and $5 \mathrm{~g} \mathrm{NaCl}$ ) or LB agar (LB containing $15 \%$ agar) were used as culture media unless otherwise indicated. Antibiotics were added, when necessary, at the following concentrations: $100 \mu \mathrm{g}$ ampicillin $\mathrm{ml}^{-1}, 50 \mu \mathrm{g}$ kanamycin $\mathrm{ml}^{-1}$ and $50 \mu \mathrm{g}$ nalidixic acid $\mathrm{ml}^{-1}$.

Recombinant DNA constructions and nucleotide sequencing. DNA restriction endonuclease digests, electrophoresis, and ligations were performed by standard procedures (Sambrook et al., 1989). Plasmids were introduced into competent cells of E. coli strains by heat-shock transformation or electroporation. Electroporations were carried out in $10 \%(\mathrm{v} / \mathrm{v})$ glycerol in $0 \cdot 1 \mathrm{~cm}$ cuvettes with an E. coli pulser (Bio-Rad) set at $1.8 \mathrm{kV}$. PCR experiments, unless otherwise stated, were performed in $100 \mu \mathrm{l}$ volumes containing $100 \mathrm{ng}$ template DNA, $0 \cdot 1 \mu \mathrm{M}$ appropriate primers, $0.2 \mathrm{mM}$ deoxynucleotide triphosphates, $1 \times$ Thermopol buffer, $4 \mathrm{mM} \mathrm{MgSO}_{4}$ and $0.5 \mathrm{U}$ DeepVent Polymerase (New England Biolabs) in accordance with the manufacturer's instructions. Reactions were subjected to initial denaturation of $2 \mathrm{~min}$ at $94^{\circ} \mathrm{C}$ followed by 29 cycles of $48^{\circ} \mathrm{C}$ for $45 \mathrm{~s}, 72^{\circ} \mathrm{C}$ for $2 \mathrm{~min}$ and $95^{\circ} \mathrm{C}$ for $1 \mathrm{~min}$.

To replace the full-length portion of $b f p A$ with $t c p A$, pLKC1 template DNA and primers Donne-36 (5'-CCAAAACGATCTCAAGAAGATCATC-3') and Donne-232 (5'-AGATCTTTTATGAAGTAAACTAGTTAATACGCCCT-3'), which encodes a UAA stop codon (bold faced) and unique BglII and SpeI linker restriction sites (italicized), were used to PCR amplify a $16 \mathrm{~kb}$ fragment containing downstream $b f p A$ 
Table 1. Bacterial strains and plasmids used in this study

\begin{tabular}{|c|c|c|}
\hline $\begin{array}{l}\text { Strain or } \\
\text { plasmid }\end{array}$ & Relevant genotype & Reference or source \\
\hline \multicolumn{3}{|l|}{ Strains } \\
\hline \multicolumn{3}{|l|}{ E. coli } \\
\hline E2348/69 & Prototype O127:H6 EPEC strain & Levine et al. (1985) \\
\hline UMD901 & $\mathrm{E} 2348 / 69$ bfpAS129C & Zhang \& Donnenberg (1996) \\
\hline UMD932 & $\mathrm{E} 2348 / 69 \Delta b f p A:: t c p A, b f p P^{\prime}:: a p h A-3::^{\prime} b f p P$ & This study \\
\hline UMD936 & $\mathrm{E} 2348 / 69 \Delta b f p A:: t c p A$ & This study \\
\hline UMD940 & $\mathrm{E} 2348 / 69 \Delta b f p A:: t c p A, b f p F^{\prime}:: a p h A-3::^{\prime} b f p F$ & This study \\
\hline $\mathrm{DH} 5 \alpha$ & $\Delta l a c U 169$ recA1 endA1 gyrA96 relA1 & Sambrook et al. (1989) \\
\hline DH5 $\alpha \lambda$ pir & $\mathrm{DH} 5 \alpha(\lambda p i r)$ & Ménard et al. (1993) \\
\hline \multicolumn{3}{|l|}{ V. cholerae } \\
\hline O395 Sm & Classical O1 Ogawa strain; $\mathrm{Sm}^{\mathrm{R}}$ & Taylor et al. (1987) \\
\hline BGD4 & $\operatorname{tcp} A$ & R. Taylor* \\
\hline \multicolumn{3}{|l|}{ Plasmids } \\
\hline pCR-Script & High-copy-number cloning vector, $\mathrm{Cml}^{\mathrm{R}}$ & Stratagene \\
\hline pCVD442 & Positive-selection suicide vector, $\mathrm{Amp}^{\mathrm{R}}$ & Donnenberg \& Kaper (1991) \\
\hline pJPN101 & EAF region II encoding $b f p A-L$ cloned into pJBK68 & Nataro et al. (1987) \\
\hline pLKC1 & pACYC184 containing $4 \cdot 2 \mathrm{~kb}$ HindIII fragment from pJPN101 & This study \\
\hline pLKCDEL1 & $\begin{array}{l}\text { pCR-Script containing } 3 \cdot 4 \mathrm{~kb} \text { PCR fragment encoding the first } 123 \\
\text { residues of BfpA and upstream sequences }\end{array}$ & This study \\
\hline pLKCDEL2 & $\begin{array}{l}\text { pCR-Script containing } 1.7 \text { PCR fragment encoding last } 11 \text { residues } \\
\text { of BfpA and downstrean } b f p \text { sequences }\end{array}$ & This study \\
\hline pLKCDEL3 & $\begin{array}{l}\text { pLKCDEL2 containing } 2 \cdot 1 \mathrm{~kb} S p e \mathrm{I}-E c o \mathrm{RV} \text { fragment from } \\
\text { pLKCDEL1; encodes in-frame } b f p A \text { deletion }\end{array}$ & This study \\
\hline pWKS30 & Low-copy-number cloning vector, $A m p^{R}$ & Wang \& Kushner (1991) \\
\hline pBPM14 & pCR-Script containing $1.6 \mathrm{~kb}$ of sequence downstream of $b f p A$ & This study \\
\hline pBPM15 & pCR-Script containing $3 \cdot 2 \mathrm{~kb}$ of sequence upstream of $b f p A$ & This study \\
\hline pBPM16 & pBPM14 containing $3 \cdot 3 \mathrm{~kb}$ NotI-BglII fragment from pBPM15 & This study \\
\hline pBPM17 & pBPM16 containing 674 bp NdeI-SpeI fragment from pBPM18 & This study \\
\hline pBPM18 & pCR-Script containing 685 bp PCR fragment encoding $t c p A$ & This study \\
\hline pBPM21 & $\begin{array}{l}\text { pCVD } 442 \text { containing } t c p A \text { flanked by } 1350 \text { bp of upstream and } \\
1050 \text { bp of downstream } b f p \text { nucleotide sequence }\end{array}$ & This study \\
\hline pBPM22 & $\begin{array}{l}\text { pCR-Script containing } 1.5 \mathrm{~kb} \text { PCR fragment encoding first } 58 \\
\text { residues of BfpA and upstream sequences }\end{array}$ & This study \\
\hline pBPM23 & $\begin{array}{l}\text { pCR-Script containing } 1.6 \mathrm{~kb} \text { PCR fragment encoding last } 156 \\
\text { residues of TcpA and downstream } b f p \text { sequences }\end{array}$ & This study \\
\hline pBPM26 & pBPM23 containing $1.6 \mathrm{~kb} K p n \mathrm{I}-S p e \mathrm{I}$ fragment from pBPM22 & This study \\
\hline pBPM27 & pWKS30 containing $1 \mathrm{~kb}$ Bam HI-PstI fragment from pBPM26 & This study \\
\hline pBPM33 & $\begin{array}{l}\text { pCR-Script containing } 211 \text { bp PCR fragment encoding } 67 \text { residues } \\
\text { of the intrachain disulfide bond region of TcpA }\end{array}$ & This study \\
\hline pBPM34 & pLKCDEL3 containing 201 bp SpeI fragment from pBPM33 & This study \\
\hline pBPM35 & pWKS30 containing $1 \mathrm{~kb} \mathrm{BamHI-PstI} \mathrm{fragment} \mathrm{from} \mathrm{pBPM34}$ & This study \\
\hline pKDS8.2 & pCVD442 containing Asp700 fragment encoding $b f p F:: a p h A-3$ & Anantha et al. (1998) \\
\hline pRPA100 & $\begin{array}{l}\mathrm{pWKS} 30 \text { containing } 1 \mathrm{~kb} \text { Bam HI-Pst } \text { fragment encoding } b f p A \\
\text { and } b f p G^{\prime}\end{array}$ & $\begin{array}{l}\text { R. Anantha, University of } \\
\text { Maryland }\end{array}$ \\
\hline
\end{tabular}

*Dept of Microbiology, Dartmouth Medical School, USA.

sequences. Similarly, primers Donne-26 (5'-AACGCGGGGAGGCAGACA-3') and Donne-233 (5'-AGATCTAACCATATGAACTGTTTTCCTTAT-3'), which encode an AUG start codon (bold faced) and unique NdeI and BglII linker restriction sites (italicized), were used to PCR amplify a $3 \cdot 2 \mathrm{~kb}$ fragment containing upstream $b f p A$ sequences. PCR products were cloned separately into pCR-Script, resulting in plasmids pBPM14 and pBPM15, respectively. To destroy the NdeI site present in $b f p B$, a 527 bp NdeI-XbaI fragment was excised from pBPM14. Both 3' recessive ends were filled in using DNA polymerase I large (Klenow) fragment and were subsequently religated. A $3 \cdot 3 \mathrm{~kb}$ Not $\mathrm{I}-$ BglII fragment excised from pBPM15 was cloned into similar sites of pBPM14, resulting in pBPM16. Additionally, a $1.4 \mathrm{~kb}$ SacI fragment was excised from pBPM16 to remove a SalI site encoded by pACYC184 sequences. Template DNA derived from $V$. cholerae was used 
with primers Donne-250 (5'-GCGGACTAGTTTAGCTGTTACCAAATGCACCGCC-3'), which encodes a UAA stop codon (bold faced) and a unique SpeI restriction site (italicized), and Donne-251 (5'-GGCGCATATGCAATTATTAAAACAGC-3'), which encodes a unique NdeI restriction site (italicized) containing an AUG start codon (bold faced), to amplify tcpA. PCR products were cloned into pCR-Script, resulting in pBPM18. A 674 bp NdeI-SpeI fragment encoding tcpA was excised from pBPM18 and cloned into similar sites of pBPM16. The resulting plasmid, pBPM17, contains approximately $1350 \mathrm{bp}$ and $1050 \mathrm{bp}$ of upstream and downstream nucleotide sequence from EPEC, respectively, flanking the complete nucleotide sequence of $t c p A$. Both pBPM17 and pCVD442 were digested with SacI, ligated and subsequently digested with SalI to remove pCR-Script vector sequences. The resultant plasmid, pBPM21, was used as a positiveselection suicide vector.

To replace sequences encoding the leader region of TcpA with those of BfpA, primers Donne-291 (5'-ACTAGTTGCGCTCATTACTTCTG-3') and Donne-294 (5'-TGAGTGTTCTGCTCACTACAG-3') were used to amplify a $1.5 \mathrm{~kb}$ fragment from $\mathrm{pLKC1}$. The resultant PCR product contained upstream $b f p$ gene cluster DNA and sequences encoding the first 58 amino acid residues of BfpA. Similarly, template DNA derived from pBPM17 was used with primers Donne-289 $\left(5^{\prime}-A C T\right.$ AGTCAAGTTGCACTGACACAGAC-3') and Don-1 (5'AGAATCACTGGAACTAC-3') to amplify a $1.6 \mathrm{~kb}$ fragment encoding the last 156 amino acid residues of $T c p A$ and remaining downstream $b f p$ sequences. The junction site chosen represents a region of similar primary and predicted secondary structure shared between both molecules. We also note that the inclusion of SpeI sites in primers Donne-289 and Donne-291 (italicized) does not change the amino acid sequence of either pilin molecule. PCR products were cloned separately into pCR-Script to create pBPM22 and pBPM23, respectively. A $1.6 \mathrm{~kb} K p n \mathrm{I}-\mathrm{SpeI}$ fragment was excised from pBPM22 and cloned into similar sites of pBPM23 to produce pBPM26. A $1 \mathrm{~kb}$ BamHI-PstI fragment was subsequently excised from pBPM26 and cloned into similar sites of the lowcopy-number vector $\mathrm{pWKS30}$ to make plasmid pBPM27.

To replace $b f p A$ sequences that encode a region inclusive of the intrachain disulfide bond of bundlin with homologous sequences derived from $t c p A$, PCR amplification was first used to construct pLKCDEL3, a plasmid that encodes an in-frame deletion of codons 123 through 183 in bundlin. Briefly, pLKC1 and primers Donne-21 and Donne-26 (5'-GCCACTAGTAAGCGTCAGCTAGTAACCAAA-3'), which contains a unique SpeI site (italicized), were used to amplify a $3.4 \mathrm{~kb}$ fragment containing sequences upstream of $b f p A$ and those encoding the first 122 amino acid residues of bundlin. Similarly, primers Donne-31 and Donne-27 (5'-GCCACTAGTACCAATAAAGTTACATAT-3'), which contains a SpeI site (italicized), were used to amplify a $1.7 \mathrm{~kb}$ fragment containing downstream $b f p$ gene cluster sequences including those that encode the last 10 amino acid residues of bundlin. Both fragments were cloned separately into pCR-Script to produce plasmids pLKCDEL1 and pLKCDEL2, respectively. A $2 \cdot 1 \mathrm{~kb} S p e \mathrm{I}-$ EcoRV fragment was excised from pLKCDEL1 and cloned into corresponding sites of pLKCDEL2 to create pLKCDEL3. Primers Donne-327 (5'-GGACTAGTTGCAAGACACTTATTACCAG-3') and Donne-311 (5'-ACTAGTACATAATTTCTCAACGTGAGTG-3') were used to amplify a $211 \mathrm{bp}$ fragment from plasmid pBPM17 and was subsequently cloned into pCR-Script. The resultant plasmid, pBPM33, encodes amino acid residues 120 through 186 of TcpA. Two SpeI sites, each of which was designed in Donne-327 and -311 (italicized), were used to excise a $201 \mathrm{bp}$ fragment from pBPM33 that was subsequently cloned into the corresponding site of pLKCDEL3 to make pBPM34. A $1 \mathrm{~kb}$ BamHI-Pst fragment was excised from pBPM34 and cloned into similar sites of pWKS30 to make pBPM35. Plasmid constructs were verified by restriction analysis and also by sequence analysis of both DNA strands using a PRISM Ready Reaction DyeDeoxy Termination kit (Applied Biosystems). Sequencing reactions were run on a model 373 DNA sequencer (Applied Biosystems) by staff of the University of Maryland Biopolymer Laboratory.

Molecular genetic techniques. The tcpA gene was first introduced into wild-type EPEC by mobilizing pBPM21 from DH5 2 ipir into E2348/69 by triparental conjugation. Allelic exchange was performed as previously described (Donnenberg et al., 1993) to produce strain UMD936. To introduce a $b f p F$ mutation into strain UMD936, plasmid pKDS82 was mobilized from DH5 $\alpha \lambda$ pir into strain UMD936 to create strain UMD940. Candidate colonies of recombinant EPEC strains possessing mutant $b f p$ alleles were verified by whole-cell PCR using fresh single colonies and Taq DNA polymerase in $100 \mu \mathrm{l}$ volumes. Reactions were subjected to initial denaturation of 2 min at $94{ }^{\circ} \mathrm{C}$ followed by 29 cycles of $54{ }^{\circ} \mathrm{C}$ for $45 \mathrm{~s}, 72{ }^{\circ} \mathrm{C}$ for $2 \mathrm{~min}$ and $95^{\circ} \mathrm{C}$ for $1 \mathrm{~min}$. Primers Donne- $5\left(5^{\prime}\right.$-AAACAGGATACCAAAAGA-3') and Donne-6 (5'-TCTTGGTGCTTGCGTGTC- $3^{\prime}$ ) were used to amplify sequences flanking $b f p A$ and for sequencing the entire tcp $A$ gene residing within the $b f p$ gene cluster from strain UMD936. Primers Donne-235 (5'-GGGAATTCCTGATTCCGTGTGATATCATG-3') and Donne-236 (5'-GGGGATCCTGCATAATATTTTAGCTAATCAGGTT-3') were used to amplify $b f p F$. Plasmid pRPA100 was electroporated into competent cells of UMD936 to complement the $b f p A$ mutation.

Tissue culture and adherence assays. HEp-2 cells (ATCC CCL 23) in monolayer culture were used as the in vitro model for EPEC adherence studies as detailed previously (Donnenberg \& Nataro, 1995). Briefly, HEp-2 cell monolayers were grown to near confluence in eight-well chamber slides (Nunc) in Eagle's minimal essential medium with $10 \%$ fetal bovine calf serum in a $5 \% \quad \mathrm{CO}_{2}$ atmosphere at $37^{\circ} \mathrm{C}$. Adherence of EPEC cultures to HEp-2 cells was performed in the presence of $1 \%$ D-mannose. Samples were examined under brightfield microscopy without prior knowledge of their identity.

Immunoblot analysis. Induction of BFP expression from EPEC was performed by inoculating $10 \mathrm{ml}$ Dulbecco's modified Eagle's medium/F-12 (DMEM/F-12) with $100 \mu$ l of LB-grown overnight cultures. DMEM/F-12 cultures were aerated (225 r.p.m.) at $37^{\circ} \mathrm{C}$ for $4-6$ h. Turbidity of each culture (measured as $\mathrm{OD}_{600}$ ) was normalized for immunoblot analysis. Induction of TCP expression from $V$. cholerae was performed as previously described (Taylor et al., 1987). Whole-cell bacterial proteins were prepared by boiling samples for $10 \mathrm{~min}$ in dissolving buffer $(625 \mathrm{mM}$ Tris $/ \mathrm{HCl}$, $\mathrm{pH} 68 ; 2 \%, \mathrm{w} / \mathrm{v}, \mathrm{SDS} ; 10 \%, \mathrm{v} / \mathrm{v}$, glycerol; $5 \%, \mathrm{v} / \mathrm{v}, 2-$ mercaptoethanol; $0.001 \%$ bromophenol blue) and subjected to SDS-PAGE $(15 \%, \mathrm{w} / \mathrm{v}$, acrylamide). Resolved proteins were electrotransferred to Immobilon-P PVDF membranes using a semidry Multiphor II NovaBlot transfer apparatus (Pharmacia) as described by the manufacturer. Membranes were blocked initially with PBS containing $01 \%(\mathrm{v} / \mathrm{v})$ Tween 20 (PBST) and $5 \%(\mathrm{w} / \mathrm{v})$ nonfat dry milk and then subjected to sequential $1 \mathrm{~h}$ incubations with either rabbit polyclonal anti-BfpA antibodies, or rabbit polyclonal anti-TcpA peptide 6 antibodies, which recognize the C-terminal portion of TcpA including the region predicted to form the intrachain disulfide 
bond (Sun et al., 1997), diluted 1:5000 in PBST, and then with goat anti-rabbit IgG antibodies (diluted 1:25000 in PBST) conjugated to alkaline phosphatase. Immunoreactive proteins were visualized using standard procedures (Harlow \& Lane, 1988).

Transmission electron microscopy. BFP were visualized by growing cells of EPEC strains in DMEM/F-12 as described above. However, cultures were subjected to brief centrifugation $(12000 \mathrm{~g}, 1 \mathrm{~min})$ and gently resuspended in $1 \mathrm{ml}$ fresh DMEM/F-12. Cultures $(10 \mu \mathrm{l})$ were spotted onto Formvarcarbon-coated copper grids, blotted, washed three times with sterile double-deionized water and stained with $1 \%(\mathrm{w} / \mathrm{v})$ phosphotungstic acid ( $\mathrm{pH} 7 \cdot 2$ ). Grids were examined on a JEOL JEM-100X transmission electron microscope.

\section{RESULTS}

\section{Construction of recombinant EPEC strains bearing full-length tcpA in place of $b f p A$}

To determine if components encoded by the $b f p$ gene cluster from EPEC can direct the assembly of hetero-

(a)

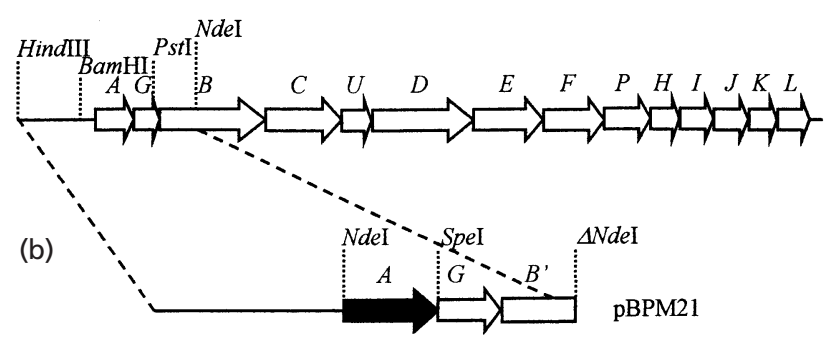

(c)

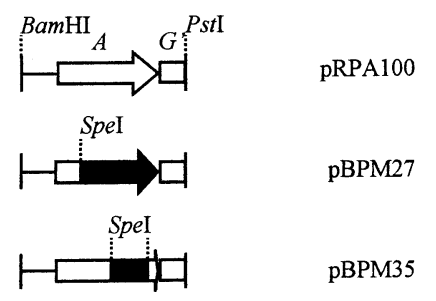

Fig. 1. Schematic representation of the bfp gene cluster from EPEC illustrating the construction of various alleles encoding type 4 pilin subunits used in this study. (a) The entire bfp gene cluster along with upstream sequences. Arrows indicate bfp genes. Selected restriction sites used for cloning are shown. (b) The expanded view illustrates the region of the bfp gene cluster used to construct strain UMD936. The positive-selection suicide-based plasmid pBPM21, which contains a region of approximately $3 \mathrm{~kb}$ including $t c p A$ sequences (denoted by the black arrow) in place of bfpA, was used for allelic exchange with the wild-type EPEC strain E2348/69. (c) Plasmid pRPA100 consists of a $1 \mathrm{~kb}$ BamHI-Pstl fragment containing $b f p A$ and $b f p G^{\prime}$ cloned into the low-copy-number plasmid pWKS30. Onekilobase BamHI-Pstl fragments containing gene fusions, which encode chimeric pilin subunits possessing either the first 58 amino acid residues of bundlin and the last 156 amino acid residues of TcpA, or bundlin subunits possessing amino acid residues 120 through 186 of $\mathrm{TcpA}$, were cloned into similar sites of pWKS30 to create plasmids pBPM27 and pBPM35, respectively. Filled areas indicate nucleotide sequences that encode corresponding regions of TcpA. logous type 4 pili encoded by $t c p A$, we amplified the entire tcpA gene from $V$. cholerae $\mathrm{O} 395$ and cloned it into the $b f p$ gene cluster in place of the entire $b f p A$ gene (Fig. 1). Plasmid pBPM17, which encodes tcp $A$ and flanking $b f p$ gene cluster sequences, was mobilized into wild-type EPEC by triparental conjugation. The sacB gene on pBPM21 was used to select for allelic exchange of full-length $t c p A$ for full-length $b f p A$, creating strain UMD936. Similarly, plasmid pKDS8.2 was mobilized into strain UMD936 as a means of introducing an aphA3-marked $b f p F$ mutation to create strain UMD940. Since cells of EPEC containing a mutation in $b f p F$ are hyperfimbriate, we hypothesized that introduction of this mutation would facilitate our ability to detect type 4 pili encoded by tcpA from EPEC. PCR analysis of wild-type EPEC, UMD936 and UMD940 confirmed the identities of $b f p A, t c p A$ and $b f p F:: a p h A-3$, respectively, based upon the expected size differences of resultant PCR products (data not shown). In addition, sequence analysis of PCR products amplified from cells of UMD936 indicated that no mutations were present in tcp $A$ and flanking sequence.

\section{EPEC strains possessing full-length tcpA fail to autoaggregate in culture and adhere poorly to cultured HEp-2 cells}

Since both BFP and TCP are required for autoaggregation of cells of EPEC and V. cholerae in culture, we tested whether TCP, if expressed and assembled on cells of UMD936 and UMD940, could also mediate formation of bacterial aggregates when cells were grown in culture media. Whereas cells of wild-type EPEC formed visible aggregates in culture, those of both recombinant strains UMD936 and UMD940, as well as UMD901, which lacks BFP due to a point mutation in $b f p A$, were incapable of autoaggregation even after extended incubation periods (data not shown). Since BFP are required for localized adherence of EPEC to host cells in vitro, we tested whether TCP could also mediate some level of adherence of bacteria to HEp-2 cells. As expected, cells of wild-type EPEC adhered in a pattern typical of localized adherence (Fig. 2a). Cells of both recombinant EPEC strains containing tcpA, like those of strain UMD901, adhered to HEp-2 cells in a manner not typical of the three-dimensional morphology associated with localized adherence (Fig. $2 \mathrm{~b}$ and data not shown).

Since the presence of $t c p A$ within the $b f p$ gene cluster could in theory have an unanticipated adverse polar effect upon downstream gene expression, we tested whether the reintroduction of $b f p A$ alone into cells of strain UMD936 could restore assembly of functional BFP. Cells of UMD936, when transformed with pRPA100 encoding wild-type $b f p A$, were capable of performing localized adherence that was indistinguishable from that of the wild-type (Fig. 2c) and formed visible bacterial aggregates when grown in culture (data not shown). As expected, the complementing plasmid pRPA100 was capable of restoring both the localized 

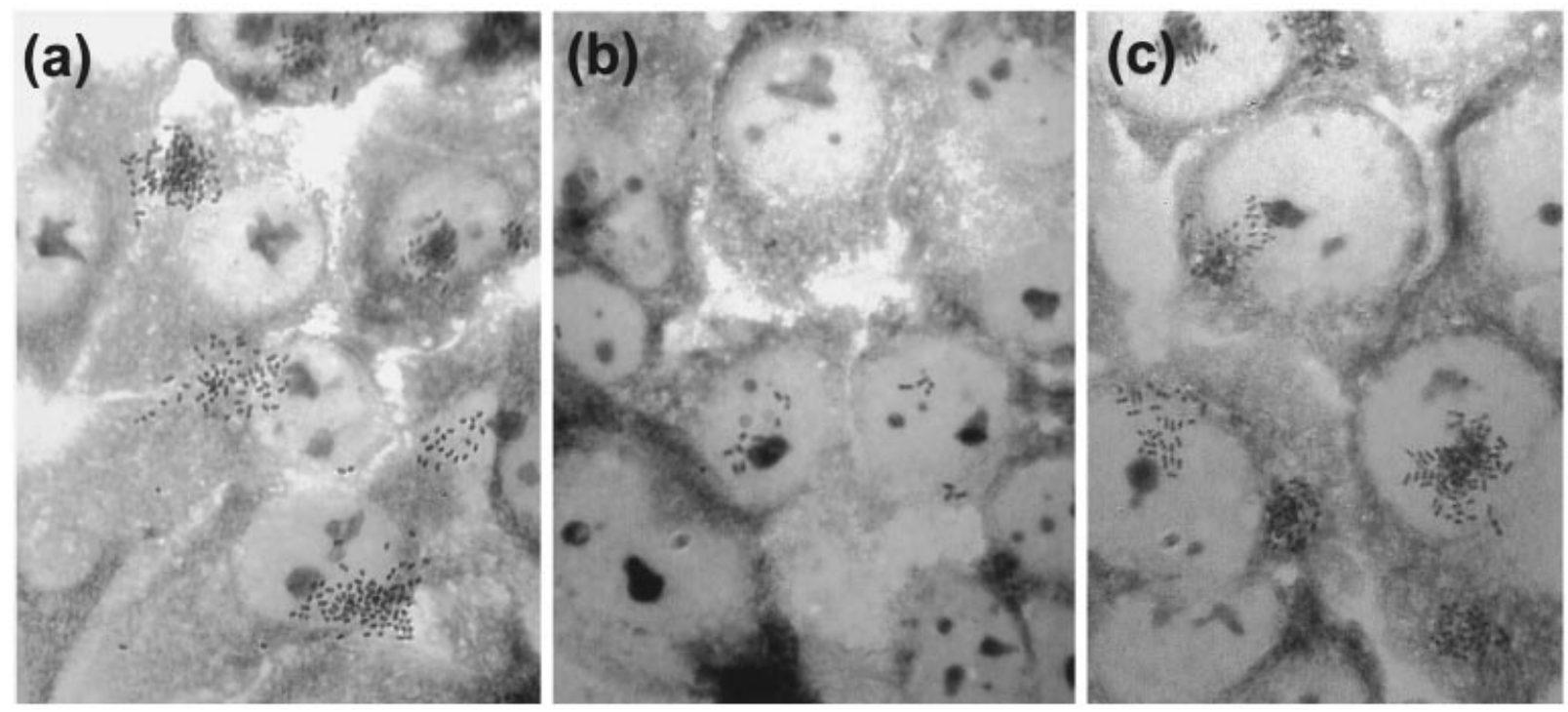

Fig. 2. Localized adherence of EPEC strains to HEp-2 cells. HEp-2 cells were incubated for $3 \mathrm{~h}$ with bacteria of the indicated strains, fixed, stained with Giemsa and examined by brightfield microscopy. (a) Wild-type EPEC strain E2348/69. (b) EPEC strain UMD936, containing tcpA in place of $b f p A$. (c) EPEC strain UMD936 transformed with pRPA100.
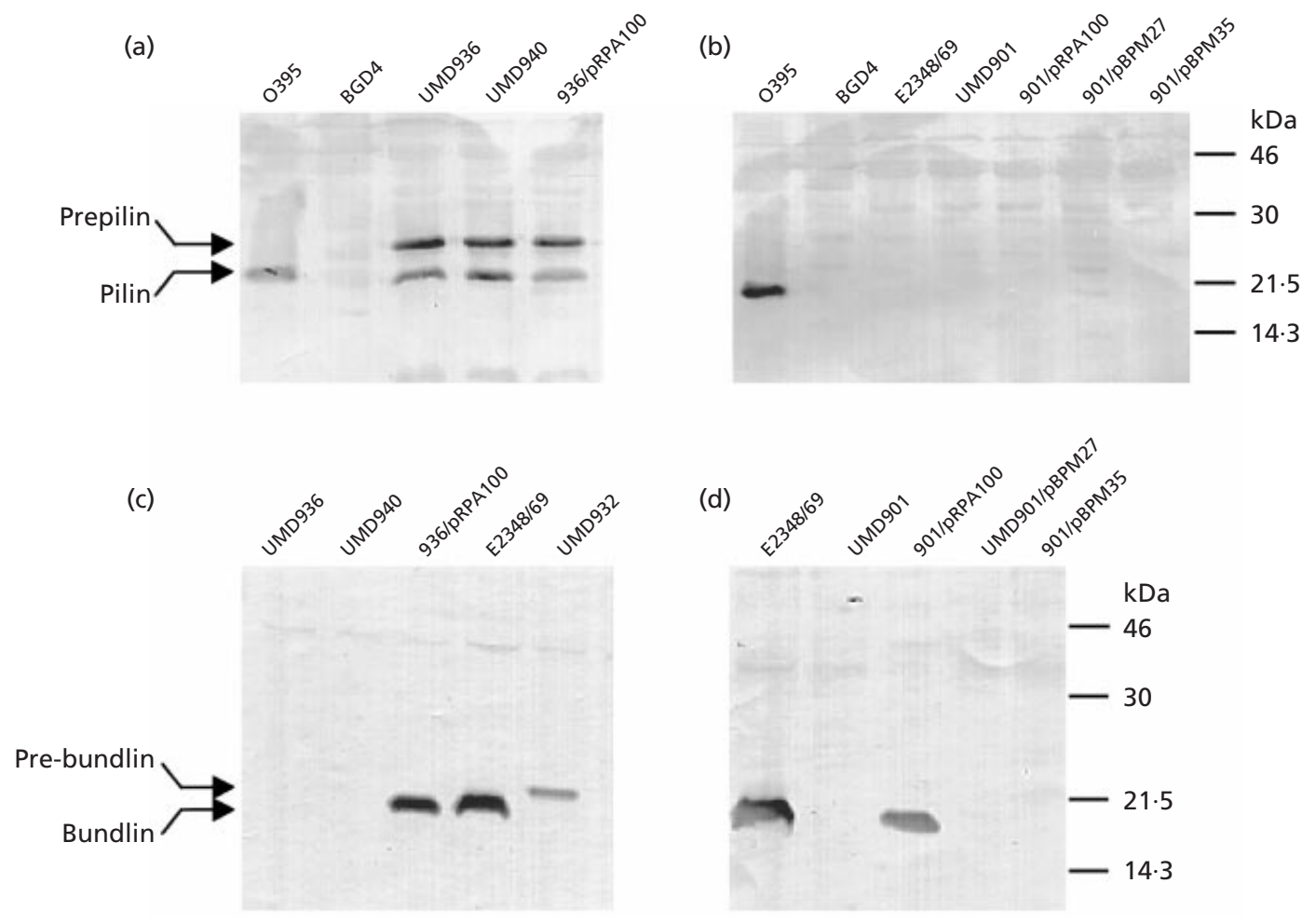

Fig. 3. Expression of various alleles encoding type 4 pilin subunits from cells of $V$. cholerae O395, $V$. cholerae strain BGD4 $(\triangle t c p A)$, wild-type EPEC E2348/69, UMD901 (bfpAS129C), UMD932 ( $\triangle$ bfpP'::aphA-3::'bfpP), UMD936 ( $\triangle b f p A:: t c p A)$ and UMD940 ( $\left.\triangle b f p A:: t c p A, b f p F^{\prime}:: a p h A-3:: b f p F\right)$. Samples from whole-cell lysates were adjusted for culture density, separated by $15 \%$ SDS-PAGE, transferred to PVDF, and probed with polyclonal rabbit antiserum that recognizes either the extreme C-terminal portion of TcpA including the region predicted to form the intrachain disulfide bond (a, b) or bundlin $(c, d)$. Arrows indicate prepilin and mature pilin proteins. The positions of molecular mass markers are indicated on the right. 
adherence and autoaggregation phenotypes in cells of UMD901 (data not shown). These results indicate that the assembly machinery encoded by the $b f p$ gene cluster in UMD936 is functionally intact and is capable of assembling BFP but not heterologous type 4 pili encoded by $t c p A$.

\section{Recombinant strains of EPEC fail to process TcpA completely}

Prepilin subunits of the type 4 pilin family must undergo proteolytic cleavage of their signal sequence before they become available for polymerization into a helical structure. Since the biogenesis of type 4 heterologous pili from EPEC would be dependent upon cleavage of unprocessed TcpA by BfpP, the endogenous prepilin peptidase, we examined the proteolytic fate of TcpA from cells of UMD936 and UMD940 by immunoblot analysis. Unlike the single mature species of TcpA that was detected in lysates derived from cells of V. cholerae, two immunoreactive proteins of approximately $23 \mathrm{kDa}$ and $20 \mathrm{kDa}$, representing unprocessed and mature forms of TcpA, respectively, were detected in lysates from both UMD936 and UMD940 (Fig. 3a). No immunoreactive product was detected from lysates of the $t c p A$ mutant $V$. cholerae strain BGD4 (Fig. 3a). To determine if the $t c p A$ gene present in the $b f p$ gene cluster affected BfpP function, we also assessed the proteolytic fate of bundlin (the $b f p A$ gene product) from cells of UMD936 transformed with pRPA100. As shown in Fig. 3(c), only a single lower-molecular-mass species representing mature bundlin was detected in lysates of wild-type EPEC and the UMD936 transformant. In contrast, a single higher-molecular-mass species representing pre-bundlin was only detected in the $b f p P$ mutant strain UMD932 (Fig. 3c). These results indicate that processing of TcpA by BfpP from cells of either UMD936 or UMD940 is incomplete. They also demonstrate that failure to fully process TcpA in these strains is not due to a defect in BfpP function since cells of UMD936 were still capable of completely processing BfpA.

\section{Recombinant strains of EPEC fail to assemble type 4 pili encoded by tcpA}

Although both recombinant EPEC strains UMD936 and UMD940 failed to process TcpA completely, the amount processed could in theory represent a large enough pool of pilin subunits available for pilus assembly. In addition, the failure of cells of either strain to form autoaggregates in culture and to adhere to HEp-2 cells could indicate that TCP are simply incapable of mediating the localized adherence and autoaggregation phenotypes in EPEC. Therefore, we examined both recombinant strains for the presence of type 4 pilin structures by negative staining and transmission electron microscopy. As demonstrated in Fig. 4(a), BFP were observed from cells of wild-type EPEC. Likewise, similar structures were consistently observed from cells of the UMD936 transformant strain containing pRPA100 (Fig. 4c) but not from those of untransformed strain UMD936

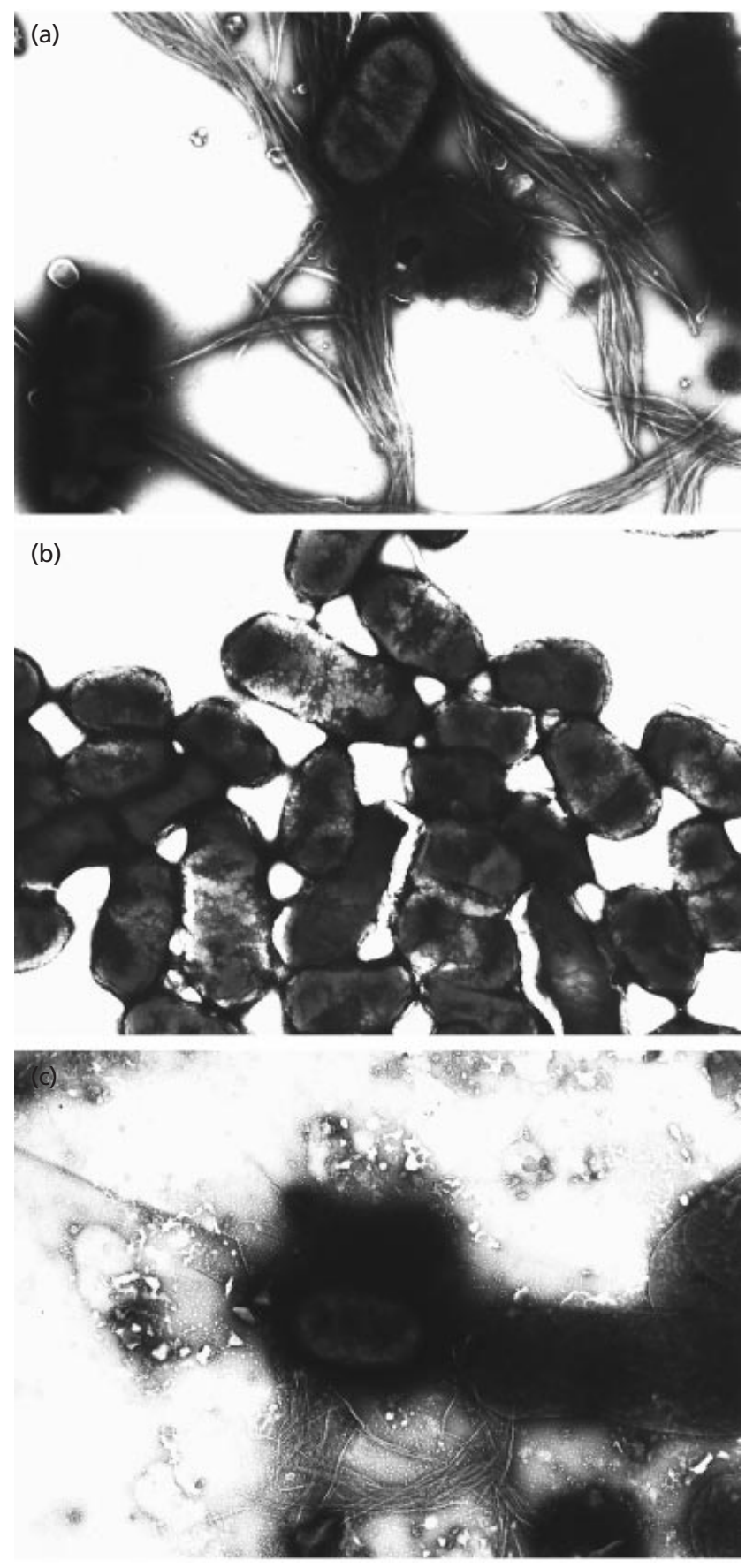

Fig. 4. Electron microscopy examination of type 4 pilus structures expressed by wild-type and recombinant EPEC strains. Strains were grown under conditions for optimal expression of BFP, spotted onto Formvar-carbon-coated copper grids, stained with phosphotungstic acid and analysed by transmission electron microscopy. (a) Wild-type EPEC strain E2348/69; (b) EPEC strain UMD936, containing tcpA in place of bfpA; (c) EPEC strain UMD936 transformed with pRPA100.

(Fig. 4b). Similarly, type 4 pilin structures were only observed from transformants of UMD901 containing pRPA100 and not from untransformed strain UMD901 or from strain UMD940 (data not shown). Furthermore, 
we did not detect evidence of TcpA export nor assembly of visible pili when we examined shear preparations from cells of strain UMD936 or when we examined recombinant cells by immunogold electron microscopy (data not shown). These results indicate that the available pool of processed TcpA pilin subunits fails to assemble into observable pilin structures. Thus, failure of both recombinant strains of EPEC to adhere to HEp2 cells in vitro and to form autoaggregates in culture is associated with the inability to express heterologous type 4 pili.

\section{Expression of chimeric type 4 pilin subunits from EPEC is not sufficient to reconstitute type 4 pilus biogenesis}

Since recombinant strains of EPEC fail to assemble TcpA subunits into a mature filament, we sought to determine if chimeric type 4 pilin subunits could be expressed and assemble in EPEC. To do so, we constructed plasmid pBPM27 to encode chimeric pilin subunits containing the first 58 amino acid residues of BfpA and the last 156 amino acid residues of TcpA (Fig. 1). Similarly, we constructed plasmid pBPM 35 to encode chimeric bundlin subunits containing a region inclusive of the intrachain disulfide bond of TcpA in order to assess whether substitution of this region would be permissive for subsequent pilin subunit recognition and filament assembly (Fig. 1). Both plasmids were constructed to be identical to the complementing plasmid pRPA100, which contains $b f p A$ and upstream promoter sequences, except for those regions that encode specific portions of TcpA in place of bundlin. Both transformant strains of UMD901, containing either pBPM27 or pBPM35, were examined for their ability to adhere to HEp-2 cells, autoaggregate, process chimeric type 4 prepilin subunits and assemble type 4 pili. Cells of either transformant adhered poorly to HEp-2 cells in a manner that was indistinguishable from that of the parent strain UMD901 (data not shown). Interestingly, neither processed nor unprocessed chimeric type 4 pilin subunits were detected in whole-cell lysates of either UMD901 transformant regardless of whether anti-TcpA (Fig. 3b) or anti-bundlin (Fig. 3d) antibodies were used. As expected, processed bundlin was detected by antibundlin antibodies from lysates of strain UMD901 transformed with pRPA100 and from wild-type EPEC (Fig. 3d). Thus, it appears that the inability of either UMD901 transformant to carry out functions mediated by type 4 pili is due to the instability of both type 4 pilin chimeric subunits.

\section{DISCUSSION}

Type 4 pili are expressed from a wide range of Gramnegative bacteria. Despite the diversity of species that possess these structures, it appears that type 4 pili represent a cohesive group that are structurally and functionally related. Conservation of the arrangement and identities of homologous genes involved in pilus assembly from various species indicates that pilin subunits are assembled by a common mechanism and suggests that they may be interchanged among compatible hosts. This is exemplified by the work of several groups who have demonstrated that $P$. aeruginosa can assemble fimbrial subunits of D. nodosus, M. bovis and N. gonorrhoeae into mature filaments on the cell surface (Watson et al., 1996; Hoyne et al., 1992; Beard et al., 1990; Mattick et al., 1987). Despite the ability of these groups to interchange various group A pilin subunits, our attempts to substitute one group B pilin subunit, BfpA from EPEC, with another, TcpA from V. cholerae, were unsuccessful. Cells of UMD936, the EPEC recombinant strain containing the full replacement of $b f p A$ with $t c p A$, consistently failed to assemble type 4 pili as judged by their failure to autoaggregate in culture, adhere to HEp-2 cells in vitro, and assemble any type 4 pilin structures. Two observations suggest that this failure was not due to the manner in which the $b f p A$ gene was replaced with tcpA in situ. First, this replacement was precise, as no other sequences within the $b f p$ gene cluster were altered other than those which encoded bundlin. Second, reintroduction of $b f p A$, encoded on pRPA100, into cells of UMD936 resulted in their ability to perform localized adherence, autoaggregate and assemble processed bundlin into mature filaments. Thus, we conclude that the assembly machinery encoded by the bfp gene cluster in UMD936 is functionally intact, but is incapable of assembling type 4 pili composed of TcpA. We also examined whether a $b f p F$ mutation would facilitate expression of type 4 pili encoded by tcpA from EPEC since cells of a $b f p F$ mutant, like those of a pilT mutant, produce many more bacterial filaments than do wild-type cells (Bieber et al., 1998; Bradley, 1974). Similar to our earlier results, cells of strain UMD940 containing bfpF and the tcpA substitution also failed to autoaggregate in culture, adhere to HEp-2 cells in vitro, and assemble any detectable surface structures resembling type 4 pili. Thus, failure to detect any type 4 pilin structures encoded by tcpA from cells of UMD936 would not necessarily be the result of our limits of detection.

EPEC and V. cholerae O395 are both enteric pathogens that express group $B$ type 4 pili. Conservation of the type 4 pilin assembly genes of these organisms suggested that EPEC would be capable of expressing type 4 pili encoded by tcpA from $V$. cholerae. Genes required for TCP or BFP biogenesis appear to be organized or clustered in a single operon (Ramer et al., 1996; Brown \& Taylor, 1995). Both gene clusters begin with the gene encoding the major structural subunit, $t c p A$ and $b f p A$, respectively; these two genes display $30.4 \%$ identity. These genes are followed by a region of dyad symmetry that is predicted to regulate expression of downstream loci and the clusters conclude with the remaining 13 genes, many of which encode proteins that share sequence similarities (Ogierman et al., 1993; Sohel et al., 1996; Stone et al., 1996). These include putative nucleotide-binding proteins $\mathrm{BfpD}$ and $\mathrm{TcpT}$; integral outer-membrane lipoproteins TcpC and BfpB; cytoplasmic-membraneassociated proteins $\mathrm{BfpE}$ and TcpE; and prepilin peptidases BfpP and TcpJ. BFP and TCP also share 
several functional similarities including the bundling of individual filaments and the formation of bacterial aggregates in culture (Taylor et al., 1987; VuopioVarkila \& Schoolnik, 1991), which may facilitate colonization of the host in vivo (Bieber et al., 1998). Despite the fact that EPEC and V. cholerae share many features of type 4 pilus biogenesis, EPEC fail to assemble pili encoded by tcpA.

Our studies indicate that the prepilin peptidases of EPEC and $V$. cholerae may not be ideal functional homologues since EPEC fail to process TcpA completely. We note that $V$. cholerae possesses two distinct prepilin peptidases, TcpJ, which is required for TcpA processing (Kaufman et al., 1991), and VcpD, which is required for assembly of the type 4 mannose-sensitive haemagglutinin (MSHA) pilus and cholera toxin secretion (Marsh \& Taylor, 1998). The presence of these two prepilin peptidases, which by themselves exhibit limited functional homology (Marsh \& Taylor, 1998; Kaufman et al., 1991), contrasts with the reciprocal functional homology that is displayed between the prepilin peptidase PilD of $P$. aeruginosa and BfpP of EPEC (Zhang et al., 1994). Thus, a higher level of specificity may be necessary for complete processing of TcpA compared to that which is necessary for complete processing of bundlin. Regardless of the possible functional differences between TcpJ and BfpP, we contend that the failure of TcpA assembly in EPEC is probably not due to the inability of BfpP to fully process TcpA. The observations reported by Strom et al. (1993) support our contention. They constructed strains of $P$. aeruginosa that contained mutations in pilD. Although some strains processed as little as $5 \%$ of prepilin proteins, they still assembled mature filaments as judged by their susceptibility to killing by the pilus-specific phage PO4. Likewise, we propose that the available amounts of fully processed TcpA in EPEC should be sufficient for subsequent assembly into mature filaments. If so, these observations suggest that constraints which limit TcpA assembly in EPEC occur after production and processing of prepilin subunits. These constraints could result from non-productive interactions occurring between TcpA and existing components encoded by the $b f p$ gene cluster, or from the failure of TcpA to engage with a specific assembly component(s) not present in EPEC. The fact that $t c p A$ in EPEC is permissive to $b f p A$ complementation in trans supports the latter mechanism.

Although similarities exist between the type 4 pili assembly systems of EPEC and V. cholerae, failure of EPEC to assemble TcpA into mature filaments may be due to several notable differences that exist between these systems. These differences include the possible requirement of a putative minor pilin subunit encoded by $t c p B$ for TCP biogenesis (Manning, 1997) that would be lacking in EPEC. EPEC may lack other functional homologues that are required for TCP assembly in $V$. cholerae. For example, TcpH, TcpQ and TcpS are all predicted to be periplasmic-associated proteins in $V$. cholerae, while only $\mathrm{BfpU}$, a highly hydrophilic protein, has been proposed to reside in the periplasm in EPEC (Stone et al., 1996; K. D. Stone \& M. S. Donnenberg, unpublished data). Interestingly, the $t c p$ gene cluster also encodes TcpI, a peptide that is highly homologous to the family of methyl-accepting chemotaxis proteins which are involved in environmental sensing (Ogierman et al., 1996); the $b f p$ gene cluster lacks such a homologue. The observation that bundlin is rapidly degraded in the absence of DsbA (Zhang \& Donnenberg, 1996), a periplasmic enzyme that mediates disulfide bond formation, contrasts with those of Peek \& Taylor (1992), who showed that TCP are still produced in a $t c p G(V$. cholerae dsbA homologue) mutant. The different effects of these disulfide isomerases on assembly of two similar type 4 pili from these two species could reflect differences in the pilin subunits, differences in the kinetics of pilus biogenesis and disulfide bond formation, or differences in periplasmic proteases that degrade malfolded pilin subunits.

To our knowledge, we have made the first attempt to express and assemble chimeric type 4 pilin subunits from an appropriate host. The first chimera, which possessed the first 58 amino acid residues of bundlin and the last 156 amino acid residues of TcpA, was designed to test whether sequences residing in the $\mathrm{N}$-terminal region of bundlin were sufficient for recognition of chimeric pilin subunits by components of the BFP machinery and, thus, would facilitate their assembly into a functional pilus. The second chimera, a bundlin subunit that contained amino acid residues 120 through 186 of TcpA in place of residues 123 through 183 of bundlin, was designed for two reasons. The first was to determine whether components of the BFP machinery would tolerate changes in a region of the pilin subunit known to contain the most divergent primary structure. The second was to determine whether this region of the chimeric pilin subunit, if assembled into a functional pilus, would confer adhesion properties similar to that of TCP. Both chimeras were carefully designed so that each junction was at equivalent portions of both molecules. Our results indicate that neither chimera was well tolerated in EPEC. Failure to detect either chimera was unlikely to be due to limitations of the antibodies that we used, since regions of either bundlin or TcpA are present in both chimeras and should be recognized by at least one of the polyclonal rabbit antisera. Likewise, failure to detect either chimera was unlikely to be due to inefficient pilin gene expression, since the nucleotide sequences of both gene fusions were identical to that of wild-type $b f p A$, encoded on pRPA100, except for those regions which encode specific portions of TcpA in place of bundlin. Thus, we conclude that failure to detect either chimeric pilin subunit in EPEC lysates is the result of the degradation of abnormal pilin proteins by specific proteases.

Collectively, our results suggest that the type 4 assembly system of EPEC exhibits some level of specificity with respect to pilin subunit recognition and assembly. Whether this is a unique feature of type 4 pili biogenesis from EPEC, reflects the limited interchangeability of 
pilin subunits belonging to group $\mathrm{B}$, or is a more general feature of type 4 pilus biogenesis systems other than Pseudomonas remains to be determined. As such, it may be difficult to use EPEC as a heterologous host for expression of type 4 pilin subunits derived from different enteric pathogens.

\section{ACKNOWLEDGEMENTS}

We thank L. Carlson for constructing plasmids pLKC1, pLKCDEL1, -2 and -3 , R. Taylor for use of the rabbit polyclonal anti-TcpA peptide 6 antibodies, and R. Anantha for the construction and use of plasmid pRPA100 and the $b f p P$ mutant strain UMD932. We also thank R. Blank and R. Anantha for their helpful comments in reviewing this manuscript. This work was supported by a Public Health Service Award AI37606.

\section{REFERENCES}

Anantha, R. P., Stone, K. D. \& Donnenberg, M. S. (1998). The role of BfpF, a member of the PilT family of putative nucleotidebinding proteins, in type IV pilus biogenesis and in interactions between enteropathogenic Escherichia coli and host cells. Infect Immun 66, 122-131.

Beard, M. K., Mattick, J. S., Moore, L. J., Mott, M. R., Marrs, C. F. \& Egerton, J. R. (1990). Morphogenetic expression of Moraxella bovis fimbriae (pili) in Pseudomonas aeruginosa. J Bacteriol 172, 2601-2607.

Bieber, D., Ramer, S. W., Wu, C. Y., Murray, W. J., Tobe, T., Fernandez, R. \& Schoolnik, G. K. (1998). Type IV pili, transient bacterial aggregates, and virulence of enteropathogenic Escherichia coli. Science 280, 2114-2118.

Bradley, D. E. (1974). The adsorption of Pseudomonas aeruginosa pilus-dependent bacteriophages to a host mutant with nonretractile pili. Virology 58, 149-163.

Brown, R. C. \& Taylor, R. K. (1995). Organization of tcp, acf, and tox $T$ genes within a ToxT-dependent operon. Mol Microbiol 16, 425-439.

Donnenberg, M. S. \& Kaper, J. B. (1991). Construction of an eae deletion mutant of enteropathogenic Escherichia coli by using a positive-selection suicide vector. Infect Immun 59, 4310-4317.

Donnenberg, M. S. \& Nataro, J. P. (1995). Methods for studying adhesion of diarrheagenic Escherichia coli. Methods Enzymol 253, 324-336.

Donnenberg, M. S., Girón, J. A., Nataro, J. P. \& Kaper, J. B. (1992). A plasmid-encoded type IV fimbrial gene of enteropathogenic Escherichia coli associated with localized adherence. Mol Microbiol 6, 3427-3437.

Donnenberg, M. S., Yu, J. \& Kaper, J. B. (1993). A second chromosomal gene necessary for intimate attachment of enteropathogenic Escherichia coli to epithelial cells. J Bacteriol 175, 4670-4680.

Girón, J. A., Levine, M. M. \& Kaper, J. B. (1994). Longus: a long pilus ultrastructure produced by human enterotoxigenic Escherichia coli. Mol Microbiol 12, 71-82.

Hahn, H. P. (1997). The type-4 pilus is the major virulenceassociated adhesin of Pseudomonas aeruginosa-a review. Gene 192, 99-108.

Harlow, D. H. \& Lane, D. (1988). Antibodies: a Laboratory Manual, Cold Spring Harbor, NY: Cold Spring Harbor Laboratory.
Herrington, D. A., Hall, R. H., Losonsky, G., Mekalanos, J. J., Taylor, R. K. \& Levine, M. M. (1988). Toxin, toxin-coregulated pili, and the tox $R$ regulon are essential for Vibrio cholerae pathogenesis in humans. J Exp Med 168, 1487-1492.

Hoyne, P. A., Haas, R., Meyer, T. F., Davies, J. K. \& Elleman, T. C. (1992). Production of Neisseria gonorrhoeae pili (fimbriae) in Pseudomonas aeruginosa. J Bacteriol 174, 7321-7327.

Johnson, K., Parker, M. L. \& Lory, S. (1986). Nucleotide sequence and transcriptional initiation site of two Pseudomonas aeruginosa pilin genes. J Biol Chem 261, 15703-15708.

Kaufman, M. R., Seyer, J. M. \& Taylor, R. K. (1991). Processing of TCP pilin by TcpJ typifies a common step intrinsic to a newly recognized pathway of extracellular protein secretion by gramnegative bacteria. Genes Dev 5, 1834-1846.

Kim, S. R. \& Komano, T. (1997). The plasmid R64 thin pilus identified as a type IV pilus. J Bacteriol 179, 3594-3603.

Koomey, M. (1995). Prepilin-like molecules in type 4 pilus biogenesis : minor subunits, chaperones or mediators of organelle translocation? Trends Microbiol 3, 409-411.

Lepper, A. W. D., Atwell, J. L., Lehrbach, P. R., Schwartzkoff, C. L., Egerton, J. R. \& Tennent, J. M. (1995). The protective efficacy of cloned Moraxella bovis pili in monovalent and multivalent vaccine formulations against experimentally induced infectious bovine keratoconjunctivitis (IBK). Vet Microbiol 45, 129-138.

Levine, M. M., Nataro, J. P., Karch, H. M., Baldini, M., Kaper, J. B., Black, R. E., Clements, M. L. \& O'Brien, A. D. (1985). The diarrheal response of humans to some classic serotypes of enteropathogenic Escherichia coli is dependent on a plasmid encoding an enteroadhesiveness factor. J Infect Dis 152, 550-559.

McKern, N. M., Stewart, D. J. \& Strike, P. M. (1988). Amino acid sequences of pilins from serologically distinct strains of Bacteroides nodosus. J Protein Chem 7, 157-164.

Manning, P. A. (1997). The tcp gene cluster of Vibrio cholerae. Gene 192, 63-70.

Marrs, C. F., Schoolnik, G., Koomey, J. M., Hardy, J., Rothbard, J. \& Falkow, S. (1985). Cloning and sequencing of a Moraxella bovis pilin gene. J Bacteriol 163, 132-139.

Marsh, J. W. \& Taylor, R. K. (1998). Identification of the Vibrio cholerae type 4 prepilin peptidase required for cholera toxin secretion and pilus formation. Mol Microbiol 29, 1481-1492.

Mattick, J. S., Bills, M. M., Anderson, B. J., Dalrymple, B., Mott, M. R. \& Egerton, J. R. (1987). Morphogenetic expression of Bacteroides nodosus fimbriae in Pseudomonas aeruginosa. J Bacteriol 169, 33-41.

Ménard, R., Sansonetti, P. J. \& Parsot, C. (1993). Nonpolar mutagenesis of the ipa genes defines IpaB, IpaC, and IpaD as effectors of Shigella flexneri entry into epithelial cells. J Bacteriol 175, 5899-5906.

Meyer, T. F., Billyard, E., Haas, R., Storzbach, S. \& So, M. (1984). Pilus genes of Neisseria gonorrheae: chromosomal organization and DNA sequence. Proc Natl Acad Sci USA 81, 6110-6114.

Nataro, J. P., Maher, K. O., Mackie, P. \& Kaper, J. B. (1987). Characterization of plasmids encoding the adherence factor of enteropathogenic Escherichia coli. Infect Immun 55, 2370-2377.

Ogierman, M. A., Zabihi, S., Mourtzios, L. \& Manning, P. A. (1993). Genetic organization and sequence of the promoter-distal region of the tcp gene cluster of Vibrio cholerae. Gene 126, 51-60.

Ogierman, M. A., Voss, E., Meaney, C., Faast, R., Attridge, S. R. \& Manning, P. A. (1996). Comparison of the promoter proximal regions of the toxin-co-regulated $t c p$ gene cluster in classical and El Tor strains of Vibrio cholerae O1. Gene 170, 9-16. 
Parge, H. E., Forest, K. T., Hickey, M. J., Christensen, D. A., Getzoff, E. D. \& Tainer, J. A. (1995). Structure of the fibre-forming protein pilin at $2.6 \AA$ resolution. Nature $378,32-38$.

Peek, J. A. \& Taylor, R. K. (1992). Characterization of a periplasmic thiol:disulfide interchange protein required for the functional maturation of secreted virulence factors of Vibrio cholerae. Proc Natl Acad Sci USA 89, 6210-6214.

Potts, W. J. \& Saunders, J. R. (1988). Nucleotide sequence of the structural gene for class I pilin from Neisseria meningitidis: homologies with the pilE locus of Neisseria gonorrhoeae. Mol Microbiol 2, 647-653.

Ramer, S. W., Bieber, D. \& Schoolnik, G. K. (1996). BfpB, an outer membrane lipoprotein required for the biogenesis of bundleforming pili in enteropathogenic Escherichia coli. J Bacteriol 178, 6555-6563.

Sambrook, J., Fritsch, E. F. \& Maniatis, T. (1989). Molecular Cloning: a Laboratory Manual, 2nd edn. Cold Spring Harbor, NY: Cold Spring Harbor Laboratory.

Shaw, C. E. \& Taylor, R. K. (1990). Vibrio cholerae O395 tcpA pilin gene sequence and comparison of predicted protein structural features to those of type 4 pilins. Infect Immun 58, 3042-3049.

Sohel, I., Puente, J. L., Murray, W. J., Vuopio-Varkila, J. \& Schoolnik, G. K. (1993). Cloning and characterization of the bundle-forming pilin gene of enteropathogenic Escherichia coli and its distribution in Salmonella serotypes. Mol Microbiol 7, 563-575.

Sohel, I., Puente, J. L., Ramer, S. W., Bieber, D., Wu, C.-Y. \& Schoolnik, G. K. (1996). Enteropathogenic Escherichia coli: identification of a gene cluster coding for bundle-forming pilus morphogenesis. J Bacteriol 178, 2613-2628.

Stone, K. D., Zhang, H.-Z., Carlson, L. K. \& Donnenberg, M. S. (1996). A cluster of fourteen genes from enteropathogenic Escherichia coli is sufficient for biogenesis of a type IV pilus. Mol Microbiol 20, 325-337.

Strom, M. S. \& Lory, S. (1993). Structure-function and biogenesis of the type IV pili. Annu Rev Microbiol 47, 565-596.

Strom, M. S., Nunn, D. N. \& Lory, S. (1993). A single bifunctional enzyme, PilD, catalyzes cleavage and $\mathrm{N}$-methylation of proteins belonging to the type IV pilin family. Proc Natl Acad Sci USA 90, 2404-2408.
Sun, D., Lafferty, M. J., Peek, J. A. \& Taylor, R. K. (1997). Domains within the Vibrio cholerae toxin coregulated pilin subunit that mediate bacterial colonization. Gene 192, 79-85.

Taniguchi, T., Fujino, Y., Yamamoto, K., Miwatani, T. \& Honda, T. (1995). Sequencing of the gene encoding the major pilin of pilus colonization factor antigen III (CFA/III) of human enterotoxigenic Escherichia coli and evidence that CFA/III is related to type IV pili. Infect Immun 63, 724-728.

Taylor, R. K., Miller, V. L., Furlong, D. B. \& Mekalanos, J. J. (1987). Use of $p h o A$ gene fusions to identify a pilus colonization factor coordinately regulated with cholera toxin. Proc Natl Acad Sci USA 84, 2833-2837.

Voss, E., Manning, P. A. \& Attridge, S. R. (1996). The toxincoregulated pilus is a colonization factor and protective antigen of Vibrio cholerae El Tor. Microb Pathog 20, 141-153.

Vuopio-Varkila, J. \& Schoolnik, G. K. (1991). Localized adherence by enteropathogenic Escherichia coli is an inducible phenotype associated with the expression of new outer membrane proteins. J Exp Med 174, 1167-1177.

Wang, R. F. \& Kushner, S. R. (1991). Construction of versatile lowcopy-number vectors for cloning, sequencing and gene expression in Escherichia coli. Gene 100, 195-199.

Watson, A. A., Mattick, J. S. \& Alm, R. A. (1996). Functional expression of heterologous type 4 fimbriae in Pseudomonas aeruginosa. Gene 175, 143-150.

Whitchurch, C. B., Hobbs, M., Livingston, S. P., Krishnapillai, V. \& Mattick, J.S. (1991). Characterisation of a Pseudomonas aeruginosa twitching motility gene and evidence for a specialised protein export system widespread in eubacteria. Gene 101, 33-44.

Zhang, H.-Z. \& Donnenberg, M. S. (1996). DsbA is required for stability of the type IV pilin of enteropathogenic Escherichia coli. Mol Microbiol 21, 787-797.

Zhang, H.-Z., Lory, S. \& Donnenberg, M. S. (1994). A plasmidencoded prepilin peptidase gene from enteropathogenic Escherichia coli. J Bacteriol 176, 6885-6891.

Received 18 May 1999; revised 27 September 1999; accepted 26 November 1999. 